\title{
Multiple View Geometry of Projector-Camera Systems from Virtual Mutual Projection
}

\author{
Shuhei Kobayashi, Fumihiko Sakaue, and Jun Sato \\ Department of Computer Science and Engineering \\ Nagoya Institute of Technology \\ Nagoya 466-8555, Japan
}

\begin{abstract}
Recently, projector camera systems have been used actively for image synthesis and for $3 \mathrm{D}$ reconstruction. For using the projector camera systems in these applications, it is very important to compute the geometry between projectors and cameras accurately. Recently, it has been shown that by using the mutual projection of cameras, multiple cameras are calibrated quite accurately. However, this property cannot be used for projector camera systems, since projectors are light-emitting devices and the projection of cameras cannot be obtained in projector images. In this paper, we show that by using the shadow of cameras and the shadow of projectors generated by projector light, we can generate virtual mutual projections between projectors and cameras, and projectors and projectors. These virtual mutual projections can be used for calibrating projector-camera systems quite accurately. Furthermore, the calibration can be achieved without using any $3 \mathrm{D}$ points unlike the existing calibration methods. The accuracy of the proposed method is evaluated by using real and synthetic images.
\end{abstract}

\section{Introduction}

In recent years, projector-camera systems have been studied extensively and used actively as new information presenting systems 24/7/5]. For using the projectorcamera systems, it is very important to calibrate cameras and projectors accurately. Since projectors can be considered as single viewpoint cameras, camera calibration methods such as the standard 8 point algorithm are often used for calibrating projector-camera systems. However, the existing methods require many 3D points for calibrating the systems accurately and stably 89]. To cope with this problem, Nishie et al. 3] proposed a useful calibration method, which uses the shadow information of objects generated by a projector light. The method enables us to calibrate projector-camera systems only from two 3D points. However, the method does not provide us good results, if these two points are close to the screen, or these two points are collinear with cameras and projectors.

On the other hand, Sato 6] showed that multiple cameras can be calibrated very accurately, if the cameras are projected to each other. It is called a mutual projection of cameras. Since projectors can be considered as single viewpoint cameras, we can expect that projector-camera systems can be calibrated accurately and reliably by using the mutual projection between projectors and 
cameras. Unfortunately, the mutual projection between projectors and cameras does not occur, since the projectors are light emitting devices and they cannot see the $3 \mathrm{D}$ world unlike cameras. Also, if we have multiple projectors, these projectors cannot see other projectors, and mutual projection between multiple projectors does not occur.

However, we in this paper show that by using the shadow of cameras and the shadow of projectors generated by the light of projectors, we can virtually generate the mutual projection between projectors and cameras, and projectors and projectors. Based on these properties, we propose a new method for calibrating projector-camera systems quite accurately from shadows of cameras and projectors. Unlike the existing calibration methods, the proposed method does not require any $3 \mathrm{D}$ point for calibrating projector-camera systems except the shadow information, and thus singular cases of the existing methods, e.g. coplanar 3D points, are no longer singular in the proposed method.

\section{Shadow Projection in Projector-Camera Systems}

The projector-camera systems consist of multiple cameras and multiple projectors. Since a projector has a single center of projection, it can be considered as a camera. Thus, the geometry between multiple projectors and multiple cameras can be analyzed by using the multiple view geometry of multiple cameras.

In general a projector projects light on a planar screen. Thus, we can consider a planar homography, $\mathbf{H}_{p c}$, between a projector and a camera with respect to the screen. The homography, $\mathbf{H}_{p c}$, can be obtained by projecting four or more than four points from the projector to the screen, and observing these points by the camera. In the following part of this paper, we assume that planar homographies between a camera and projectors are available.

Let us consider a $3 \mathrm{D}$ point, $\mathbf{X}$, a projector, $\mathbf{P}$, and a camera, $\mathbf{C}$, as shown in Fig. 1. Because of a light of the projector $\mathbf{P}$, a shadow $\mathbf{X}^{\prime}$ of the point $\mathbf{X}$ is generated on a screen $\Pi$. Let $\mathbf{X}$ and $\mathbf{X}^{\prime}$ be projected to $\mathbf{x}$ and $\mathbf{x}^{\prime}$ in an image of the camera $\mathbf{C}$. Also, let $\mathbf{x}_{p}$ be a point in the projector image, which corresponds to the $3 \mathrm{D}$ point $\mathbf{X}$. Then, the relationship between $\mathbf{x}_{p}$ and $\mathbf{x}^{\prime}$ can be described by using the planar homography $\mathbf{H}_{p c}$ as follows:

$$
\mathbf{x}^{\prime} \sim \mathbf{H}_{p c} \mathbf{x}_{p}
$$

where, $\sim$ denotes an equality up to a scale, and point coordinates are represented by using homogeneous coordinates.

Now, if we consider a projection matrix $\mathbf{A}$ of the camera $\mathbf{C}$ and a projection matrix $\mathbf{A}^{p}$ of the projector $\mathbf{P}$, the $3 \mathrm{D}$ point $\mathbf{X}$ is projected to $\mathbf{x}$ and $\mathbf{x}_{p}$ as follows:

$$
\begin{aligned}
\mathbf{X} & \sim \mathbf{A X} \\
\mathbf{x}_{p} & \sim \mathbf{A}^{p} \mathbf{X}
\end{aligned}
$$

Thus, the $3 \mathrm{D}$ point $\mathbf{X}$ is projected to the projection, $\mathbf{x}^{\prime}$, of the shadow point, $\mathbf{X}^{\prime}$, as follows:

$$
\mathbf{x}^{\prime} \sim \mathbf{A}^{\prime} \mathbf{X}
$$




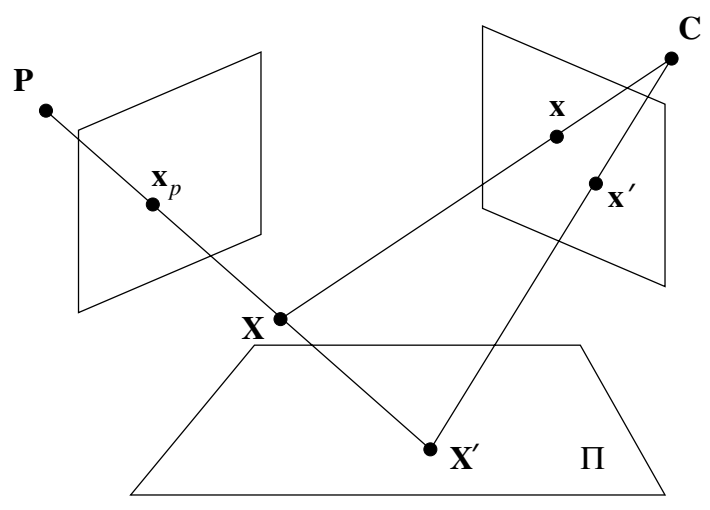

Fig. 1. The shadow projection in a projector-camera system which consists of a projector and a camera

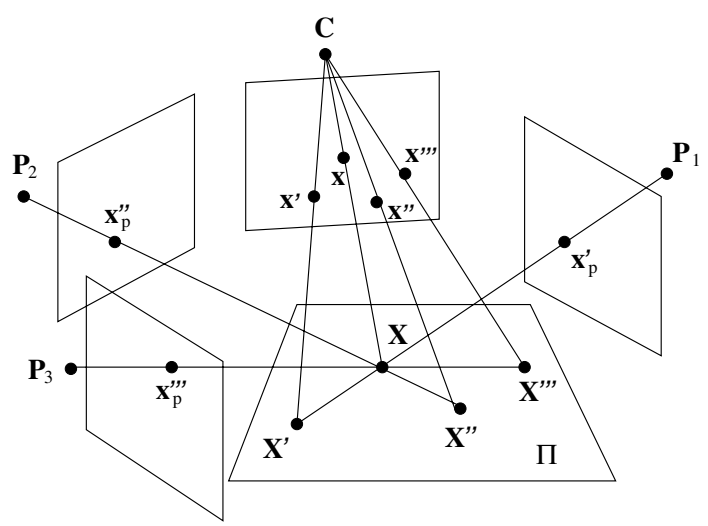

Fig. 2. The shadow projection in a projector-camera system which consists of three projectors and a camera

where, $\mathbf{A}^{\prime}$ is the following projection matrix:

$$
\mathbf{A}^{\prime}=\mathbf{H}_{p c} \mathbf{A}^{p}
$$

From (2) and (41), we find that the projection, $\mathbf{x}$, of $\mathbf{X}$ and the projection, $\mathbf{x}^{\prime}$, of its shadow $\mathbf{X}^{\prime}$ are considered as a pair of stereo camera images, and thus $\mathbf{A}$ and $\mathbf{A}^{\prime}$ are projection matrices of a pair of stereo cameras. Since the relationship between $\mathbf{A}^{\prime}$ and $\mathbf{A}^{p}$ can be described by using a planar homography $\mathbf{H}_{p c}$ as shown in (5), $\mathbf{A}^{\prime}$ and $\mathbf{A}^{p}$ are projectively equivalent. Thus, $\mathbf{A}^{\prime}$ is considered as a projection matrix of the projector $\mathbf{P}$ up to a projective ambiguity. Thus, two view geometry between $\mathbf{A}$ and $\mathbf{A}^{p}$ is equivalent to the two view geometry between $\mathbf{A}$ and $\mathbf{A}^{\prime}$ up to a projective ambiguity.

The above discussions can be hold for projector camera systems with two or more than two projectors. Suppose we have a camera and three projectors 
as shown in Fig. 2, and three shadows $\mathbf{X}^{\prime}, \mathbf{X}^{\prime \prime}$ and $\mathbf{X}^{\prime \prime \prime}$ of a $3 \mathrm{D}$ point $\mathbf{X}$ are generated by these three projectors. These shadows are observed as $\mathbf{x}^{\prime}, \mathbf{x}^{\prime \prime}$ and $\mathbf{x}^{\prime \prime \prime}$ in the camera image. Then the relationships between the $3 \mathrm{D}$ point $\mathbf{X}$ and image points can be described by using projection matrices as follows:

$$
\begin{aligned}
\mathbf{X} & \sim \mathbf{A} \mathbf{X} \\
\mathbf{x}^{\prime} & \sim \mathbf{A}^{\prime} \mathbf{X} \\
\mathbf{x}^{\prime \prime} & \sim \mathbf{A}^{\prime \prime} \mathbf{X} \\
\mathbf{x}^{\prime \prime \prime} & \sim \mathbf{A}^{\prime \prime \prime} \mathbf{X}
\end{aligned}
$$

From (6), (7), (8), and (9), we find that $\mathbf{A}^{\prime}, \mathbf{A}^{\prime \prime}$ and $\mathbf{A}^{\prime \prime \prime}$ can be considered as projection matrices of the three projectors up to a projective ambiguity, and thus we can consider the multiple view geometry of multiple projectors and a camera by using the projections of shadow points.

\section{Virtual Mutual Projection of Projectors and Cameras}

We next consider virtual mutual projections between projectors and cameras, and projectors and projectors. Sato [6] showed that if multiple cameras are projected to each other, then the multiple view geometry of these cameras can be computed more reliably from less corresponding points. The mutual projection occurs between two cameras. However, it does not occur between a projector and a camera, since the projector is a light emitting device, and it cannot observe the camera. Also, the mutual projection between a projector and another projector does not occur. However, if we use shadow information caused by the projector light, we can virtually generate mutual projection between a projector and a camera, and a projector and another projector.

Let us consider a camera $\mathbf{C}$ and three projectors $\mathbf{P}_{1}, \mathbf{P}_{2}$ and $\mathbf{P}_{3}$. Let $\mathbf{C}^{\prime}, \mathbf{C}^{\prime \prime}$ and $\mathbf{C}^{\prime \prime \prime}$ be shadows of the camera $\mathbf{C}$ generated by the projector light of $\mathbf{P}_{1}, \mathbf{P}_{2}$ and $\mathbf{P}_{3}$ respectively. Also, let $\mathbf{X}_{i j}$ be a shadow of $\mathbf{P}_{j}$ generated by the projector light of $\mathbf{P}_{i}$. These shadows $\mathbf{C}^{\prime}, \mathbf{C}^{\prime \prime}, \mathbf{C}^{\prime \prime \prime}, \mathbf{X}_{12}, \mathbf{X}_{13}$ and $\mathbf{X}_{23}$ are projected to the camera image as $\mathbf{e}^{\prime}, \mathbf{e}^{\prime \prime}, \mathbf{e}^{\prime \prime \prime}, \mathbf{e}_{12}^{\prime}, \mathbf{e}_{13}^{\prime}$ and $\mathbf{e}_{23}^{\prime}$ respectively, and are visible in the camera image.

Then, $\mathbf{C}, \mathbf{P}_{1}$ and $\mathbf{e}^{\prime}$ are collinear as shown in Fig. 3. Therefore, $\mathbf{e}^{\prime}$ is an epipole of $\mathbf{P}_{1}$ in the image of $\mathbf{C}$. Also, $\mathbf{e}^{\prime \prime}$ and $\mathbf{e}^{\prime \prime \prime}$ are epipoles of $\mathbf{P}_{2}$ and $\mathbf{P}_{3}$ in the image of $\mathbf{C}$. On the other hand, $\mathbf{e}_{12}^{\prime}$ is the projection of $\mathbf{X}_{12}$ which is collinear with $\mathbf{P}_{1}$ and $\mathbf{P}_{2}$. This means $\mathbf{e}_{12}^{\prime}$ is the indirect observation of an epipole $\mathbf{e}_{12}$ between $\mathbf{P}_{1}$ and $\mathbf{P}_{2}$. Also, $\mathbf{e}_{13}^{\prime}$ and $\mathbf{e}_{23}^{\prime}$ are the indirect observation of epipole $\mathbf{e}_{13}$ of $\mathbf{P}_{1}$ and $\mathbf{P}_{3}$, and epipole $\mathbf{e}_{23}$ of $\mathbf{P}_{2}$ and $\mathbf{P}_{3}$ respectively. The relationship between $\mathbf{e}_{i j}$ and $\mathbf{e}_{i j}^{\prime}$ can be described by using planar homographies between the camera and projectors as follows:

$$
\begin{aligned}
& \mathbf{e}_{12} \sim \mathbf{H}_{c 1} \mathbf{e}_{12}^{\prime} \\
& \mathbf{e}_{13} \sim \mathbf{H}_{c 1} \mathbf{e}_{13}^{\prime} \\
& \mathbf{e}_{23} \sim \mathbf{H}_{c 2} \mathbf{e}_{23}^{\prime}
\end{aligned}
$$




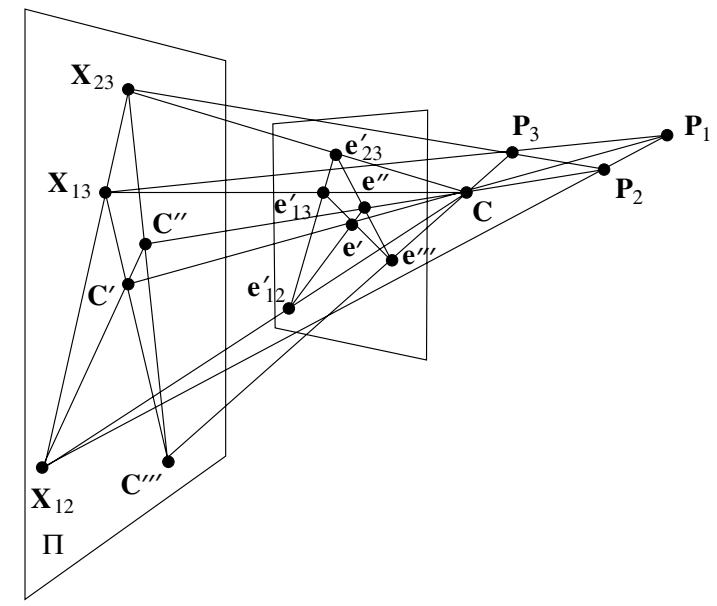

Fig. 3. Virtual Mutual Projection between cameras and projectors

where, $\mathbf{H}_{c 1}$ and $\mathbf{H}_{c 2}$ denote planar homographies between the camera and projector $\mathbf{P}_{1}$, and the camera and projector $\mathbf{P}_{2}$ respectively.

Thus, by observing the shadows of a camera, $\mathbf{e}^{\prime}, \mathbf{e}^{\prime \prime}, \mathbf{e}^{\prime \prime}$, and the shadows of projectors, $\mathbf{e}_{12}^{\prime}, \mathbf{e}_{13}^{\prime}, \mathbf{e}_{23}^{\prime}$, we can directly extract all the epipoles in the multiple view geometry of the projector-camera system. This means by observing the shadows of the camera and the projectors in the camera image, we can generate mutual projection between projectors and cameras virtually.

In the next section, we show a method for computing the multiple view geometry of projector-camera systems by using the virtual mutual projection.

\section{Multiple View Geometry from Virtual Mutual Projection}

\subsection{View Geometry}

Let us first consider the multiple view geometry of a projector-camera system which consists of a projector, $\mathbf{P}_{1}$, and a camera, $\mathbf{C}$, as shown in Fig. 4 . The light of the projector $\mathbf{P}_{1}$ generates the shadow $\mathbf{X}^{\prime}$ of a $3 \mathrm{D}$ point $\mathbf{X}$ and the shadow $\mathbf{C}^{\prime}$ of the camera $\mathbf{C}$ on the screen. Then, $\mathbf{X}, \mathbf{X}^{\prime}$ and $\mathbf{C}^{\prime}$ are projected to $\mathbf{x}=\left[x^{1}, x^{2}, x^{3}\right]^{\top}, \mathbf{x}^{\prime}=\left[x^{\prime 1}, x^{\prime 2}, x^{\prime 3}\right]^{\top}$ and $\mathbf{e}^{\prime}=\left[e^{\prime 1}, e^{\prime 2}, e^{\prime 3}\right]^{\top}$ in the camera image. Since $\mathbf{x}, \mathbf{x}^{\prime}$ and $\mathbf{e}^{\prime}$ are collinear as shown in Fig. 4. the auto-epipolar holds and the relationship between $\mathbf{x}$ and $\mathbf{x}^{\prime}$ can be described as follows:

$$
x^{i} x^{j j} e^{\prime k} \epsilon_{i j k}=0
$$

where, $\epsilon_{i j k}$ denotes a tensor whose value is 1 for even permutation, -1 for odd permutation and 0 for the other case. In this paper, the Einstein's summation convention is used for describing tensor equations. 


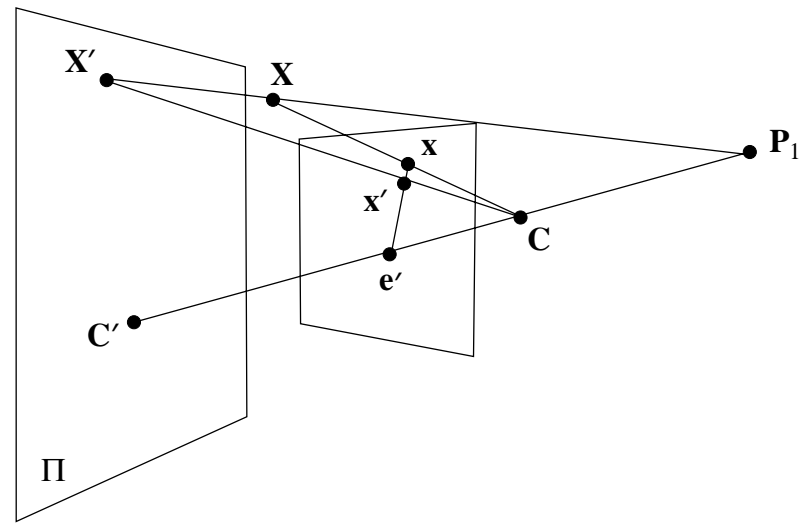

Fig. 4. Two view geometry of a projector-camera system

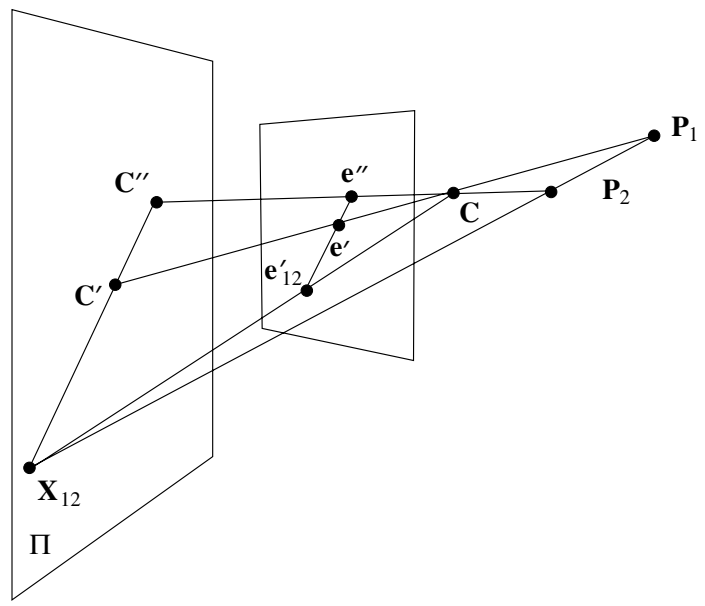

Fig. 5. Three view geometry of a projector-camera system

As shown in (13), the two view geometry of projector-camera systems can be described by using just an epipole, $\mathbf{e}^{\prime}$, i.e. the projection of the shadow of camera $\mathbf{C}$ in its own image. Thus, we no longer need any corresponding points for computing the two view geometry of a projector-camera system except a shadow of the camera.

\subsection{View Geometry}

We next consider the three view geometry of a projector-camera system, which consists of two projectors, $\mathbf{P}_{1}$ and $\mathbf{P}_{2}$, and a camera, $\mathbf{C}$, as shown in Fig. 5

Let $\mathbf{e}^{\prime}$ and $\mathbf{e}^{\prime \prime}$ be the projections of shadow $\mathbf{C}^{\prime}$ and shadow $\mathbf{C}^{\prime \prime}$, which are generated by the projector light of $\mathbf{P}_{1}$ and $\mathbf{P}_{2}$ respectively. The shadows $\mathbf{X}^{\prime}$ and $\mathbf{X}^{\prime \prime}$ 
of a $3 \mathrm{D}$ point $\mathbf{X}$ are generated by the projector light of $\mathbf{P}_{1}$ and $\mathbf{P}_{2}$ respectively. Suppose $\mathbf{X}, \mathbf{X}^{\prime}$ and $\mathbf{X}^{\prime \prime}$ are projected to $\mathbf{x}, \mathbf{x}^{\prime}$ and $\mathbf{x}^{\prime \prime}$ in the camera image. Then, the auto-epipolar holds as two view geometry, and thus, the projection matrix $\mathbf{A}$ of camera $\mathbf{C}$, the projection matrix $\mathbf{A}^{\prime}$ of projector $\mathbf{P}_{1}$ and the projection matrix $\mathbf{A}^{\prime \prime}$ of projector $\mathbf{P}_{2}$ can be described by using $\mathbf{e}^{\prime}$ and $\mathbf{e}^{\prime \prime}$ as follows:

$$
\mathbf{A}=\left[\begin{array}{ll}
\mathbf{I} & 0
\end{array}\right], \mathbf{A}^{\prime}=\left[\mathbf{I}-\mathbf{e}^{\prime}\right], \mathbf{A}^{\prime \prime}=\left[\mathbf{I}-\alpha \mathbf{e}^{\prime \prime}\right]
$$

where, $\alpha$ denotes a relative distance of $\mathbf{P}_{2}$ with respect to the projective frame defined by $\mathbf{C}$ and $\mathbf{P}_{1}$. The relative scale $\alpha$ can be described by using an epipole, $\mathbf{e}_{12}^{\prime}$, between projector $\mathbf{P}_{1}$ and projector $\mathbf{P}_{2}$ and epipoles $\mathbf{e}^{\prime}$ and $\mathbf{e}^{\prime \prime}$ as follows:

$$
e_{12}^{i}=e^{i}-\alpha e^{\prime \prime i}
$$

Thus, if we have $\mathbf{e}^{\prime}, \mathbf{e}^{\prime \prime}$ and $\mathbf{e}_{12}$, then we can compute $\alpha$ from (15).

Since the projection matrices of the camera and the projectors are as shown in (14), the trilinear relationship between $\mathbf{x}, \mathbf{x}^{\prime}$ and $\mathbf{x}^{\prime \prime}$ can be described as follows:

$$
x^{i} x^{\prime j} x^{\prime \prime k} \epsilon_{j q u} \epsilon_{k i v} e^{\prime q}-\alpha x^{i} x^{\prime j} x^{\prime \prime k} \epsilon_{j i u} \epsilon_{k r v} e^{\prime \prime r}=0_{u v}
$$

This is the minimal representation of the three view geometry of a projectorcamera system.

From (15) and (16), we find that the three view geometry of a projectorcamera system can be derived just from the shadows of the camera, $\mathbf{e}^{\prime}$ and $\mathbf{e}^{\prime \prime}$, and the shadow of the projector, $\mathbf{e}_{12}^{\prime}$, in the camera image. We do not need any corresponding point except these shadows for computing the three view geometry of a projector-camera system.

\subsection{View Geometry}

We next consider a projector-camera system which consists of three projectors, $\mathbf{P}_{1}, \mathbf{P}_{2}$ and $\mathbf{P}_{3}$, and a camera, $\mathbf{C}$, as shown in Fig. 3.

Let $\mathbf{e}^{\prime}, \mathbf{e}^{\prime \prime}$ and $\mathbf{e}^{\prime \prime \prime}$ be the projections of the shadows of camera $\mathbf{C}$, which are generated by the projector light of $\mathbf{P}_{1}, \mathbf{P}_{2}$ and $\mathbf{P}_{3}$. Also, let $\mathbf{e}_{i j}^{\prime}$ be the projection of the shadow of $\mathbf{P}_{j}$ generated by the projector light of $\mathbf{P}_{i}$. Suppose the projector light of $\mathbf{P}_{1}, \mathbf{P}_{2}$ and $\mathbf{P}_{3}$ generate shadows $\mathbf{X}^{\prime}, \mathbf{X}^{\prime \prime}$ and $\mathbf{X}^{\prime \prime \prime}$ of a $3 \mathrm{D}$ point $\mathbf{X}$, and these points are projected to $\mathbf{x}^{\prime}, \mathbf{x}^{\prime \prime}, \mathbf{x}^{\prime \prime \prime}$ and $\mathbf{x}$ in the camera image. Then, because of the auto-epipolar, the projection matrix $\mathbf{A}$ of camera $\mathbf{C}$ and the projection matrices $\mathbf{A}^{\prime}, \mathbf{A}^{\prime \prime}$ and $\mathbf{A}^{\prime \prime \prime}$ of projector $\mathbf{P}_{1}, \mathbf{P}_{2}$ and $\mathbf{P}_{3}$ can be described as follows:

$$
\mathbf{A}=[\mathbf{I} 0], \mathbf{A}^{\prime}=\left[\mathbf{I}-\mathbf{e}^{\prime}\right], \mathbf{A}^{\prime \prime}=\left[\mathbf{I}-\alpha \mathbf{e}^{\prime \prime}\right], \mathbf{A}^{\prime \prime \prime}=\left[\mathbf{I}-\beta \mathbf{e}^{\prime \prime \prime}\right]
$$

where, $\alpha$ and $\beta$ are relative distance of $\mathbf{P}_{2}$ and $\mathbf{P}_{3}$ with respect to the projective frame defined by $\mathbf{C}$ and $\mathbf{P}_{1}$, and can be computed from the following relationship between $\alpha, \beta$ and epipoles:

$$
e_{12}^{\prime i}=e^{\prime i}-\alpha e^{\prime \prime i}, e_{13}^{\prime i}=\beta e^{\prime \prime \prime} i
$$


From (17) we find that the following quadrilinear relationship holds for $\mathbf{x}, \mathbf{x}^{\prime}$, $\mathbf{x}^{\prime \prime}$ and $\mathbf{x}^{\prime \prime \prime}$ :

$$
x^{i} x^{\prime j} x^{\prime \prime k} x^{\prime \prime \prime l} \epsilon_{i p a} \epsilon_{j q b} \epsilon_{k r c} \epsilon_{l s d}\left(-\epsilon^{r s p} e^{\prime q}+\alpha \epsilon^{s p q} e^{\prime \prime r}-\beta \epsilon^{p q r} e^{\prime \prime \prime} s\right)=0_{a b c d}
$$

This is the minimal representation of the four view geometry of a projectorcamera system. Since $\alpha$ and $\beta$ can be computed from epipoles, the four view geometry can be computed just from the shadows of the camera and the shadows of the projectors. We do not need any other corresponding point in the camera image except these shadows for calibrating the projector-camera systems. Thus, projector-camera systems can be calibrated without using any $3 \mathrm{D}$ points in the scene.

\section{Experiments}

\subsection{Real Image Experiments}

In this section, we show the results from real image experiments. In these experiments, we synthesized shadows which should be generated by the projector light of arbitrary projectors by using multilinear relationships computed from the shadow of cameras and projectors.

Fig. 6] shows a projector-camera system used in our experiments. It consists of three projectors and a camera. The shadows of arbitrary projectors are generated from the trilinear relationship and the quadrilinear relationship computed from shadow information. In the experiment of trilinear relationship, we used two of these three projectors. The projector 1, 2 and 3 project red, green and blue light respectively, and the shadows caused by these projectors are separated by using color information.

Fig. 7 (a) shows a camera image obtained in the experiment of three view geometry. The epipole 1 and 2 in this figure show the center of shadows of the camera generated by projector 1 and 2 respectively. These epipoles were

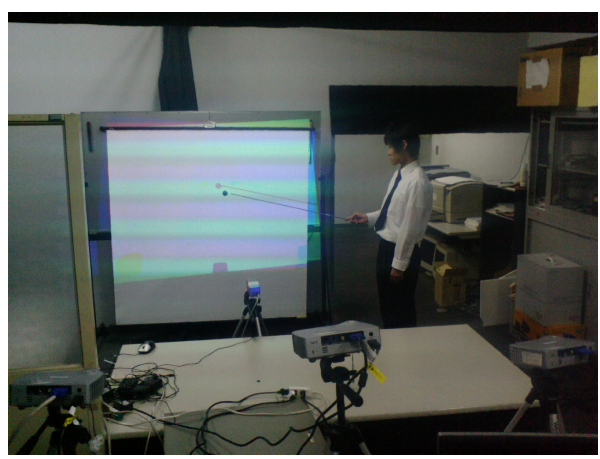

Fig. 6. Experimental set up of a projector-camera system 


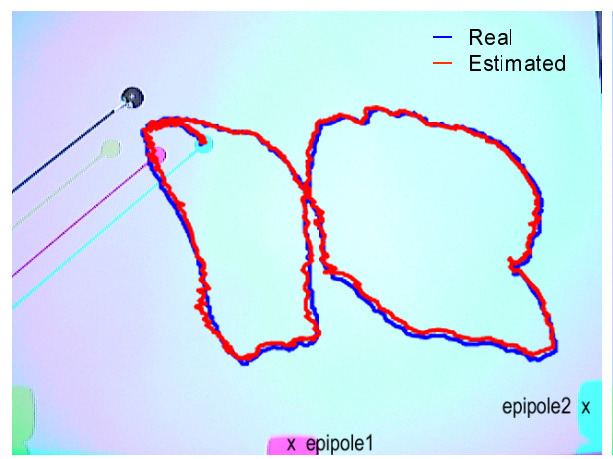

(a) three view geometry

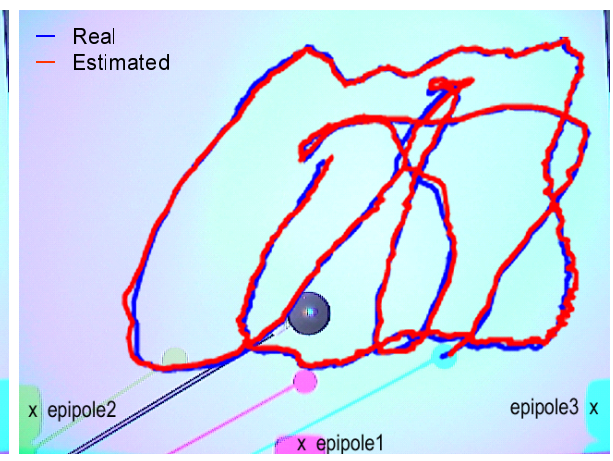

(b) four view geometry

Fig. 7. The real shadows and the shadows generated by the proposed method

extracted manually. The shadows caused by projector 2 were generated by using the trilinear relationship computed from the proposed method. The red curve shows shadow generated from the proposed method, and the blue curve shows the real shadow. As shown in this figure, the shadow of an arbitrary projector is generated properly by using the proposed method. Fig. 7 (b) shows the result from the four view geometry extracted from the proposed method. Again, the shadow generated from the proposed method is accurate.

\subsection{Stability Evaluations}

We next evaluate the stability of the proposed method. In this evaluation, bifocal, trifocal and quadrifocal tensors are computed from the proposed virtual mutual projection method, and image points are transferred from one view to another view by using the estimated multifocal tensors. The image noises with the standard deviation of 1.0 are added to all the image points. The RMS errors are computed between the estimated image points and the real image points. The results are compared with those from Nishie's method [3]. The Nishie's method requires at least two 3D points for computing multifocal tensors, while the proposed does not require any $3 \mathrm{D}$ point. If these $3 \mathrm{D}$ points are close to the screen, Nishie's method is close to singular, and it is unstable. Thus, we compare these two methods changing the distance between the screen and the $3 \mathrm{D}$ points. Since we cannot transfer image points by using bifocal tensors, we evaluated the epipolar distance, i.e. the distance between a transferred point and an epipolar line, for the two view case.

Fig. 8 shows the RMS errors derived from the estimated bifocal tensors. The horizontal axis shows the distance between the screen and the 3D points used in Nishie's method. The red line shows the result from the proposed method and the blue line shows that from Nishie's method. As shown in this graph, Nishie's method is unstable if the $3 \mathrm{D}$ points are close to the screen, while the proposed method does not require any $3 \mathrm{D}$ point and is stable all the time. 


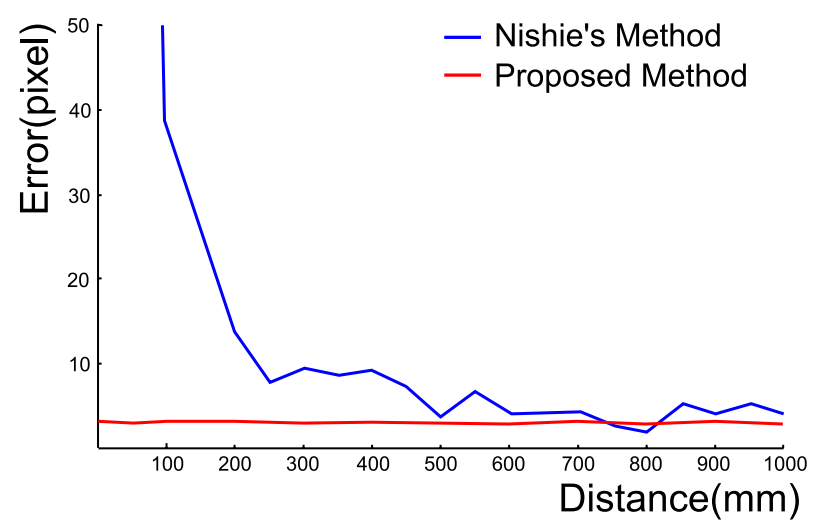

Fig. 8. Stability evaluation in the two view geometry

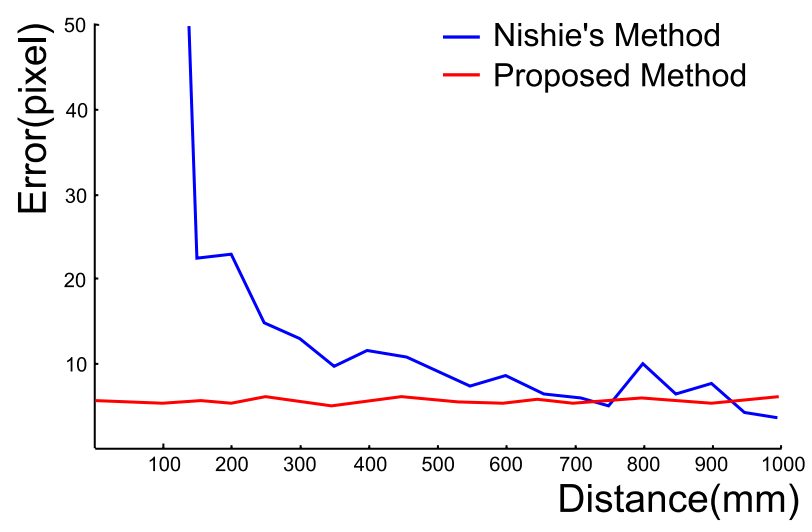

Fig. 9. Stability evaluation in the three view geometry

Fig. 9] and Fig. 10 show results from the three view case and the four view case respectively. As shown in these graphs, the stability of the proposed method is again better than Nishie's method. Since the stability of Nishie's method [3] is much better than that of traditional 8 point algorithm [1, the proposed method is quite stable and is useful for calibrating projector-camera systems.

\subsection{Accuracy of Estimated Epipoles}

We next evaluate the accuracy of epipoles estimated as the center of gravity of shadows. If the distance between the camera and the projector is large, the shadow of camera is small, and the epipole estimated from the shadow is close to the ground truth. Thus, we evaluated the relationship between the distance from the camera to the projector and the error of estimated epipoles of a camera. The result is shown in Fig. 11 (a). We also evaluated the error of epipoles of a 


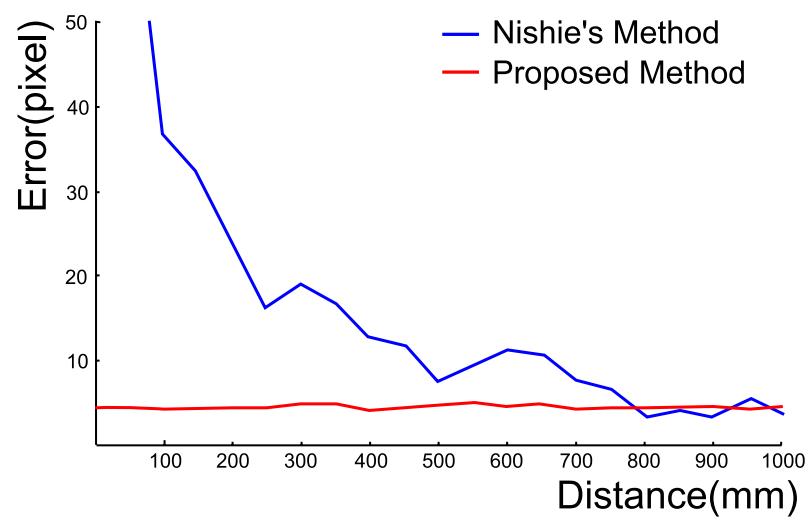

Fig. 10. Stability evaluation in the four view geometry

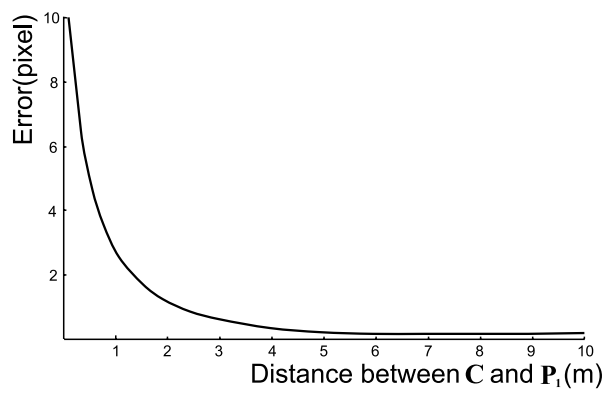

(a) epipole of camera

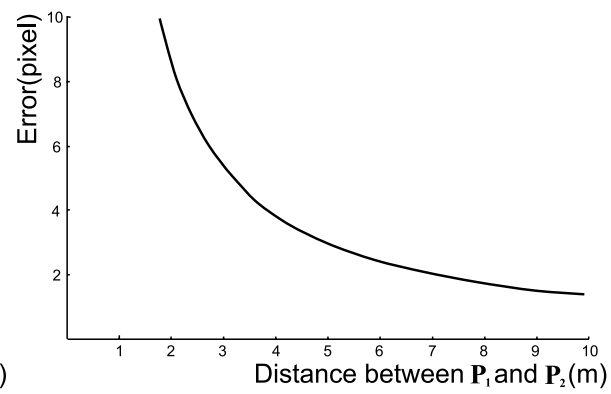

(b) epipole of projector

Fig. 11. Error of estimated epipoles

projector, changing the distance between two projectors. Fig. 11 (b) shows the result of the evaluation.

\section{Conclusion}

In this paper, we proposed a method for calibrating projector-camera systems by using mutual projections of projectors and cameras. In general mutual projections between projectors and cameras do not occur, since projectors are light emitting devices and they can not see the 3D world unlike cameras. However, we in this paper showed that by using the shadow of cameras and the shadow of projectors generated by projector lights, we can generate virtual mutual projections between projectors and cameras, and projectors and projectors. These virtual mutual projections enable us to compute the multiple view geometry of projector-camera systems and calibrate them quite accurately. Furthermore, the proposed method does not require any $3 \mathrm{D}$ points for calibrating projector-camera systems, while the traditional 8 point algorithm requires 
minimum of 8 points and Nishie's method requires minimum of 2 points.. The excellent stability of the proposed method was shown in the experiments.

\section{References}

1. Hartley, R., Zisserman, A.: Mutiple View Geometry in Computer Vision. Cambridge University Press, Cambridge (2000)

2. Milgram, P., Kishino, F.: A taxonomy of mixed reality visual display. IEICE Transactions on Information and System E77-D(12), 1321-1329 (1994)

3. Nishie, K., Sato, J.: 3D reconstruction from uncalibrated cameras and uncalibrated projectors from shadows. In: Proc. International Conference on Pattern Recognition, vol. 1, pp. 15-18 (2006)

4. Oka, K., Sato, I., Nakanishi, Y., Sato, Y., Koike, H.: Interaction for entertainment contents based on direct manipulation with bare hands. In: Proc. IWEC, pp. 397 404 (2002)

5. Okatani, T., Deguchi, K.: Autocalibration of a projector-camera system. IEEE Trans. PAMI 27(12), 1845-1855 (2005)

6. Sato, J.: Recovering multiple view geometry from mutual projections of multiple cameras. International Journal of Computer Vision 66(2), 123-140 (2006)

7. Sukuthankar, R., Stockton, R., Mullin, M.: Smarter presentations: Exploiting homography in camera-projector systems. In: Proc. ICCV 2001 (2001)

8. Raskar, R., Beardsley, P.: A self-correcting projector. In: Proc. Computer Vision and Pattern Recognition (2001)

9. Griesser, A., Van Gool, L.: Automatic Interactive Calibration of Multi-ProjectorCamera Systems. In: Proc. Conference on Computer Vision and Pattern Recognition Workshop (2006) 